http://www.jfas.info

\title{
ELECTROCARDIOGRAM NOISE CANCELLATION USING WAVELET TRANSFORM
}

F. R. Hashim ${ }^{1, *}$, J. Adnan $^{1}$, N. G. Nk Daud ${ }^{1}$, A. S. N. Mokhtar ${ }^{1}$, A. F. Rashidi ${ }^{1}$ and Z. I. $\operatorname{Rizman}^{2}$

${ }^{1}$ Department of Electrical and Electronic Engineering, Faculty of Engineering, Universiti Pertahanan Nasional Malaysia, Sg. Besi Camp, 57000 Kuala Lumpur, Malaysia ${ }^{2}$ Faculty of Electrical Engineering, Universiti Teknologi MARA, 23000 Dungun, Terengganu, Malaysia

Published online: 10 September 2017

\begin{abstract}
This paper presenting a new electrocardiogram (ECG) noise removal based on wavelet transform technique. The system involves two stages filtration, which denoising process starts with ECG thresholds with several threshold methods. The residual signal will feed to the second stage filtration using the high/low wavelet based filter. Wavelet transform methods capable to counter the unpredictability of motion artifact noise which produce by physical movement. Wavelets may reduce the motion artifact effects since it has the capability to filter both high and low frequency signals and produce good results for both stationary and non-stationary signals. The performance of the wavelet arrangement is shown by using ECG signals taken from the MIT-BIH ECG database.
\end{abstract}

Keywords: wavelet; electrocardiogram (ECG); motion artifact.

Author Correspondence, e-mail: fakroul@upnm.edu.my

doi: http://dx.doi.org/10.4314/jfas.v9i3s.11 


\section{INTRODUCTION}

Research based on the noise removal in the electrocardiogram (ECG) signals have been done by many researches. In [12] stated that the most common sources of noise contaminating the ECG signal includes Baseline Wander (BW), Powerline Interference (PLI), Electromyogram (EMG) [15] and Motion Artifact (MA). Motion artifact is the most difficult noise to be removed since frequencies of the ECG signal and the noise are overlaps. In [3] designed a new technique in order to detect the P, QRS and T peaks. They used Haar mother wavelet for QRS wave detection, while the rest $\mathrm{P}$ and $\mathrm{T}$ peak detection were done by using Daubachies mother wavelet. In [6-7] in their research used discrete wavelet transform (DWT) technique to reduce the MA noise. They used high-pass/low-pass filter to reduce the high frequency effects and the low frequency effects form ECG signal.

In [11] came with a new method, which used transform based with hybrid stage. By using the method, the ECG signal is compressed by using Discrete Cosine Transform (DCT) and combined with 1D or 2D DWT. These method is suitable for ECG compressing as well as QRS complex. In the research, QRS complex detection shows better result than the P and T peaks. The QRS complex increase in compression ratio and lower in percent root mean square difference for $1 \mathrm{D}$ and 2D hybrid technique respectively. In [1] in his study used a method based on stationary wavelet approximation in reducing the BW noise effect. In their study, the method used is non-supervised which is the main advantage compared to other techniques by allowing an automatic analysis of ECG. By using the proposed method, an accurate result is given in eliminating BW noise. They used the SWT approach based on Daubechies type 2 at level 8 . The selection of Daubechies as the mother wavelet is capable to provide good results. In the work, Daubechies type 2 approaches used, but the use of Daubechies type 4 were seen to be more similar with the ECG signal shape.

The similarity between mother wavelet and ECG signal allows maintaining the originality of signals during the noise filtering process. In $[2,13]$ have been using a combination of wavelet denoising, a FFT bandpass filter and a Bayesian filter in filtering the BW and the results are compared with conventional ECG denoising approaches. In this study, they used Coiflet type 5 as the mother wavelet but this is no better than Duabechies mother wavelet. Here, the 
similarity between mother wavelet and ECG signal plays a big role to maintain the initial signal. ECG signals corrupted with noise from subjects with Atrial Fibrillation (AF) and Left Ventricle Event (LVE) condition from the MIT-BIH library are used in this research.

\section{METHODOLOGY}

Signals from subjects with AF and LVE condition have been corrupted with motion artifact noise. The signals used were recorded using a 3-lead Holter monitor, which were augmented vector foot (aVF), augmented vector left (aVL) and augmented vector right (aVR) attached to a human body shown in Fig. 1. Several wavelet threshold methods can be used in removing the noise from signal based on two major threshold scheme, which are hard thresholding and soft thresholding. The hard threshold can be illustrated as the process of setting to zero the elements whose absolute values are lower than the threshold. The soft threshold is an extension of hard threshold, first setting to zero the elements whose absolute values are lower than the threshold and then shrinking the nonzero coefficients toward zero [10].

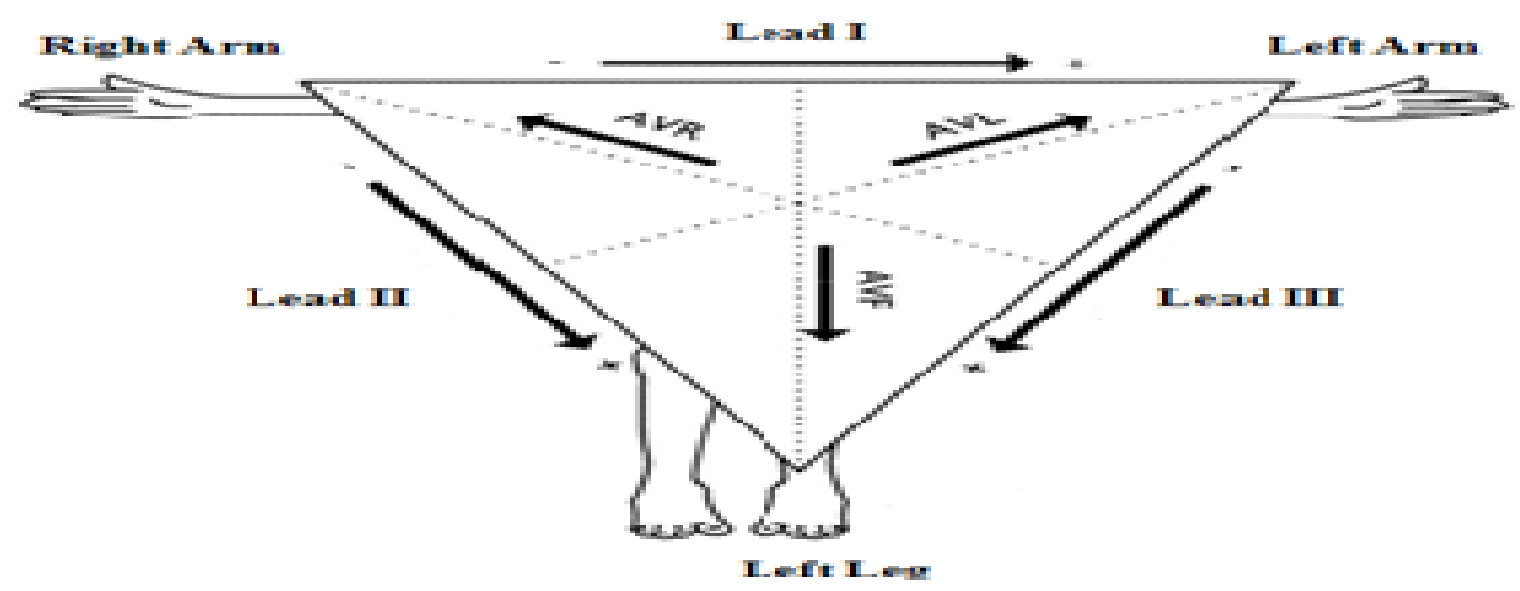

Fig.1. Leads placed on the patient's body

In signal denoising wavelet based threshold method often be used such as adaptive threshold selection using the principle of Stein's Unbiased Risk Estimate (Rigrsure), fixed threshold methods $\sqrt{ }(2 \log (\mathrm{x}))$, (Stwolog), fixed threshold heuristic variant of the first option (Heursure) and fixed threshold minimax (minimax) [9, 14].

The minimax method uses a selected fixed threshold that can produce minimax performance of the mean squared error compared to the ideal procedure. Therefore, this minimax principle can be used to design estimators for statistical applications. The minimax estimator is being 
used since it is able to obtain the minimum and maximum mean square error (MSE). The technique used in Rigrsure is using a soft threshold estimator as threshold selection for the rules, which are made on the basis of Stein's Unbiased Risk Estimate. The technique is able to estimate the risk and simultaneously minimize the risk in setting the threshold. The Sqtwolog estimator applies a fixed-form threshold technique in order to deliver minimax results and has been multiplied with a small factor proportional to log (length (signal)). The Heursure estimator is a combination between the Sqtwolog and Rigrsure estimators. Heursure depends on the value of the SNR, where very small SNR indicates the occurrence of the very noisy condition. If this situation is detected, a fixed form threshold is used to remove the noise [4-5, $8]$.

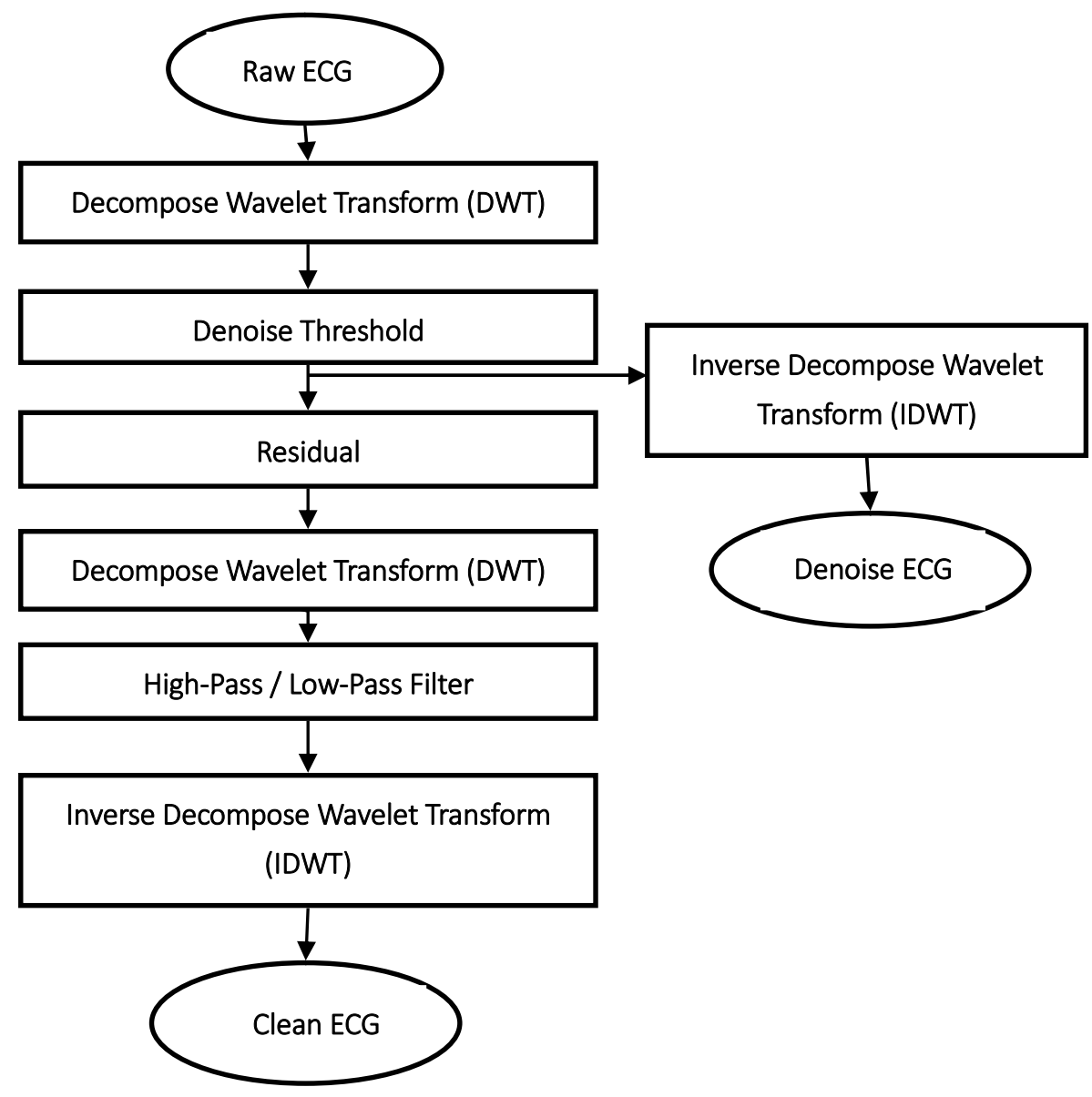

Fig.2. Block diagram for motion artifact noise removal

\section{RESULTS AND DISCUSSION}

Several methods were tested on the subjects with AF and LVE conditions. The approach involved wavelet based denoising methods (Sqtwolog, Rigrsure, Heursure and Minimax). 
Since the noise occurring in the ECG signal was unpredictable, then the wavelet based denoising is followed by the high-pass/low-pass wavelet based filtering process. The combination of these filters is successful to cancel both the low and high frequency noise. The combinations of Sqtwolog, Rigrsure, Heursure and Minimax techniques with high-pass/low-pass filter are known as Sqtwolog-HL, Rigrsure-HL, Heursure-HL and Minimax-HL respectively. The results of all methods used are shown in Table 1 for AF and Table 2 for LVE condition based on Lead aVR-aVF.

Table 1. Noise removal from normal signals based on Lead aVR-aVF

\begin{tabular}{cc}
\hline Filtering Technique & SNR of Leads Placed (Db) \\
\hline Corrupted signal & -0.91 \\
Sqtwolog & 18.75 \\
Rigrsure & 17.57 \\
Heursure & 17.56 \\
Minimax & 16.98 \\
Sqtwolog-HL & 8.12 \\
Rigrsure-HL & 7.85 \\
Heursure-HL & 7.83 \\
Minimax-HL & 6.98 \\
\hline
\end{tabular}

Table 1 shows the performance of the wavelet based filters in order to reduce the noise effect in normal ECG condition. The Sqtwolog-HL method show the best results to be compared with other wavelet based filter techniques. The results show that the wavelet denoise technique gives better result than the corrupted signal, however by combining with the wavelet based high-pass/low-pass filters have increase the filtration performance.

Based on the Table 1, the wavelet based denoising techniques (Sqtwolog, Rigrsure, Heursure and Minimax) capable to produce better results compared to the corrupted signal. However, better results are given by combining the filtration technique with wavelet based high-pass/low-pass filter. Significant improvement has been shown compared to the corrupted signals and the results obtained by using wavelet based denoising method itself. Nevertheless, the Sqtwolog-HL method shows the best performance compared to Rigrsure-HL, 
Heursure-HL and Minimax-HL in removing the noise from ECG signals as tabled in Table 1.

Based on the Table 1, small improvement has been shows by Sqtwolog, Rigrsure, Heursure and Minimax denoise techniques. The improvement range between 0.6 to $1.4 \mathrm{~dB}$. However, huge improvement has been shows after the residual signal from denoise technique been filtered with waelet based high pass/low pass filter. The improvement has been shows is up to $13 \mathrm{~dB}$.

Table 2. Noise removal from LVE signals based on Lead aVR-aVF

Filtering Technique SNR of Leads Placed (Db)

\begin{tabular}{cc}
\hline Corrupted signal & -0.50 \\
Sqtwolog & 0.09 \\
Rigrsure & -0.33 \\
Heursure & -0.32 \\
Minimax & -0.07 \\
Sqtwolog-HL & 12.98 \\
Rigrsure-HL & 11.97 \\
Heursure-HL & 11.95 \\
Minimax-HL & 12.52 \\
\hline
\end{tabular}

Table 2 exhibit the LVE signal filtering, but using wavelet based denoise threshold and high pass/low pass filter. The best results by using the Sqtwolog-HL method is used to compared with filtering result/technique with other methods. The results show that the combination of wavelet based denoising with the wavelet based high-pass/low-pass filters gives better performance compared to denoising approach alone. Based on the Table 2, the wavelet based denoising techniques (Sqtwolog, Rigrsure, Heursure and Minimax) capable to produce better results compared to the corrupted signal. However, more significant results are shows from the combining of the wavelet based denosing with wavelet based high-pass/low-pass filter. Significant improvement has been shown compared to the corrupted signals and the results obtained by using wavelet based denoising method itself. Nevertheless, the Sqtwolog-HL method shows the best performance compared to Rigrsure-HL, Heursure-HL and Minimax-HL in removing the noise from ECG signals as tabled in Table 1. 
Although using the denoising techniques alone can obtain better performance compared to corrupted signal, but the filtered signal contains with both high and low frequency noises. In order to overcome this problem, the Sqtwolog-HL method has been used since it attempts to remove both low and high frequency noise whilst simultaneously retaining the important information from the ECG signal. Fig. 3 shows the comparison between the two phases denoising process and the performance. Fig. 3(a) shows the ECG signal corrupted with noises which contains low and high frequency noise. The wavelet based denoise method (Sqtwolog) was applied to the signal and it the high frequency noise has significantly removed from the signal and leaves the low frequency noise (and some high frequency noise). As shown in Fig. 2(b). Fig. 2(c), it shows the result with the combination of wavelet based denoise method with the wavelet based high-pass/low-pass filter (Sqtwolog-HL) in removing both low and high frequency noise, leaving the clean ECG signal behind.

Nevertheless, the processed clean ECG signal is not totally clean because there is still some noise in the signal with most of the important information of the signal yet to be processed. Since the MA noise is an unpredictable signal, it is difficult to be removed. Moreover, the MA might have overlapped with ECG signal. The removal of the MA may remove the important information of ECG simultaneously.

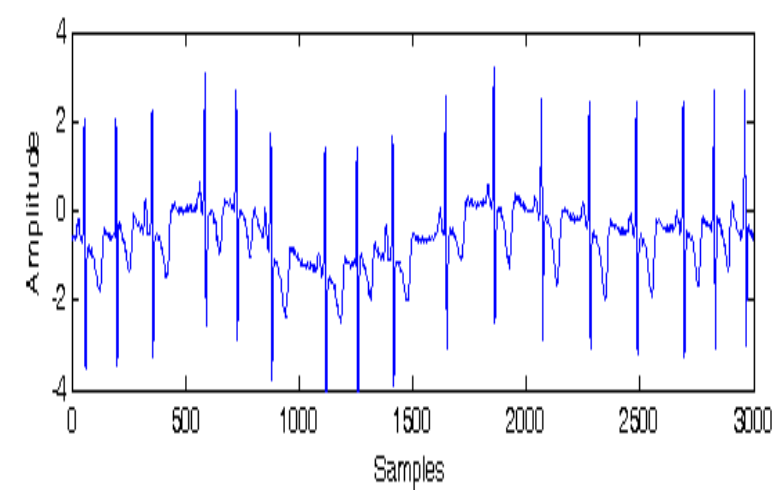

(a)

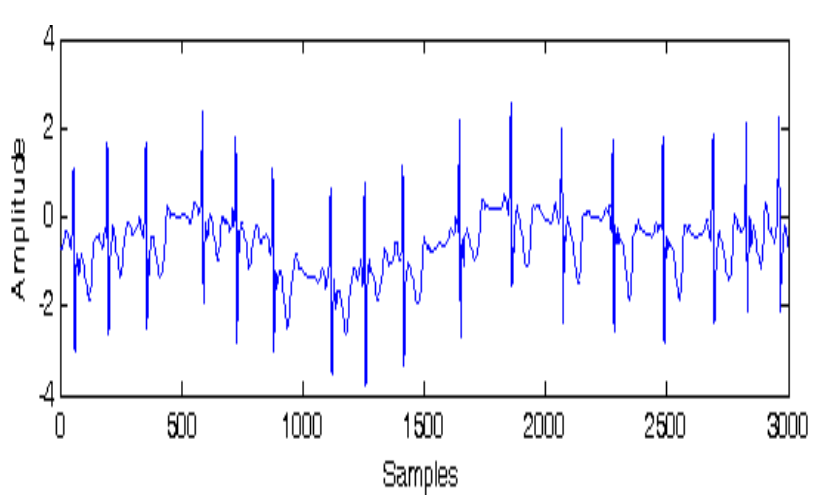

(b) 


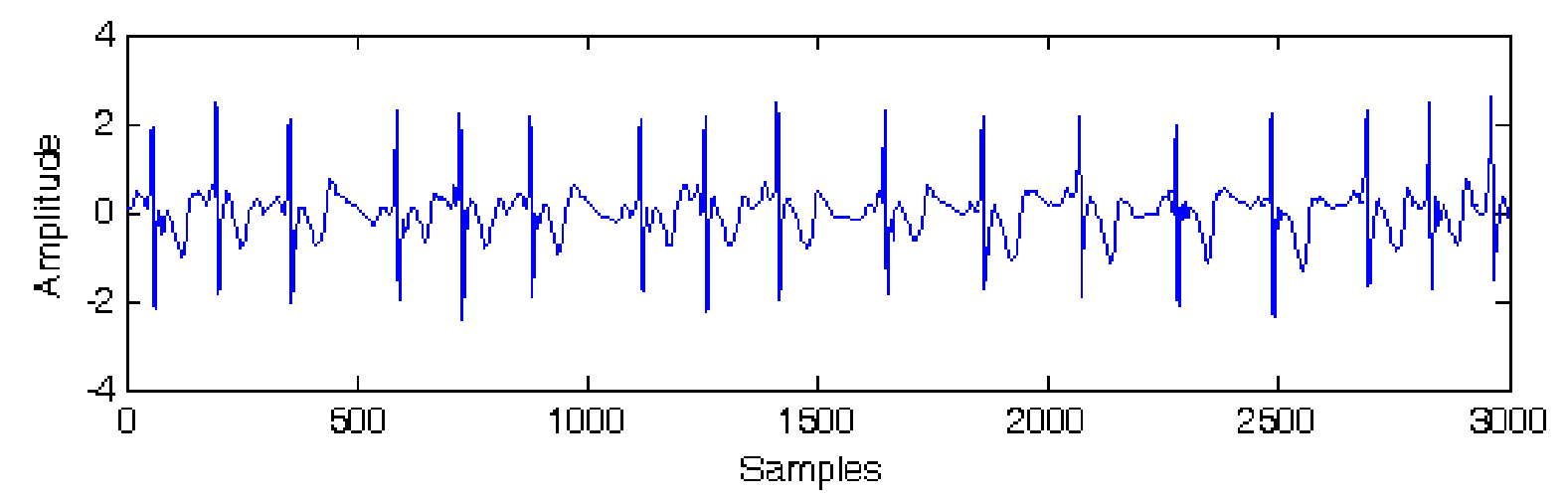

(c)

Fig.3. Result of noise removal from AF signal; (a) ECG signal with noise, (b) noise removal using Sqtwolog, (c) noise reduction using Sqtwolog-HL

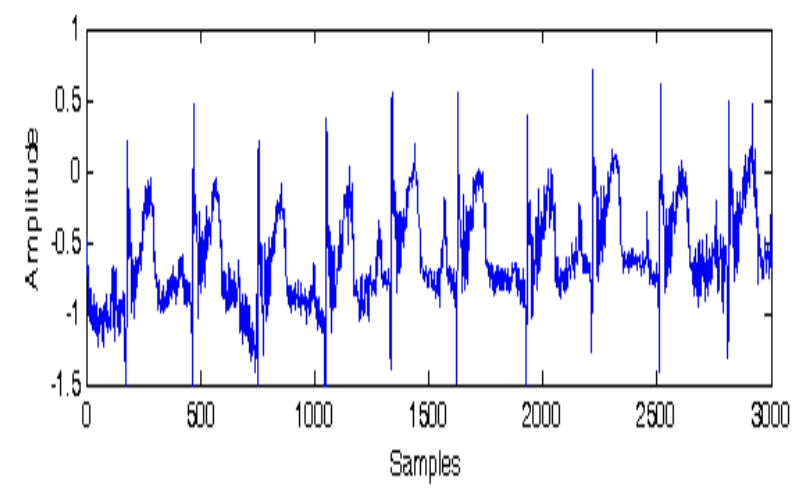

(a)

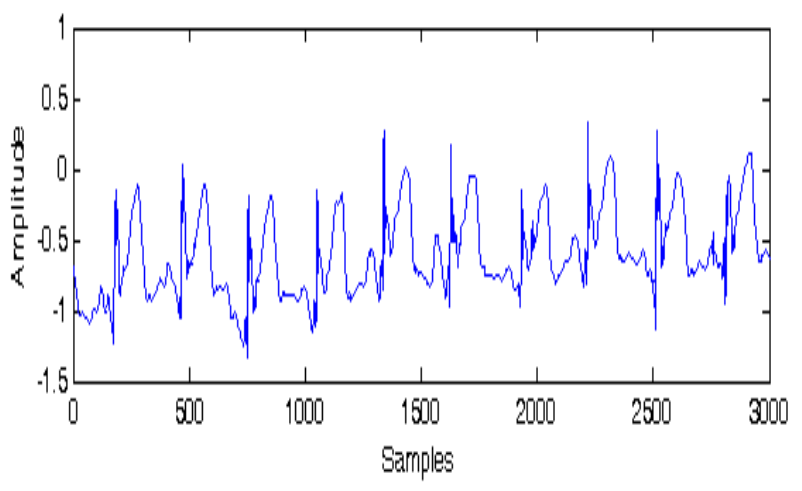

(b)

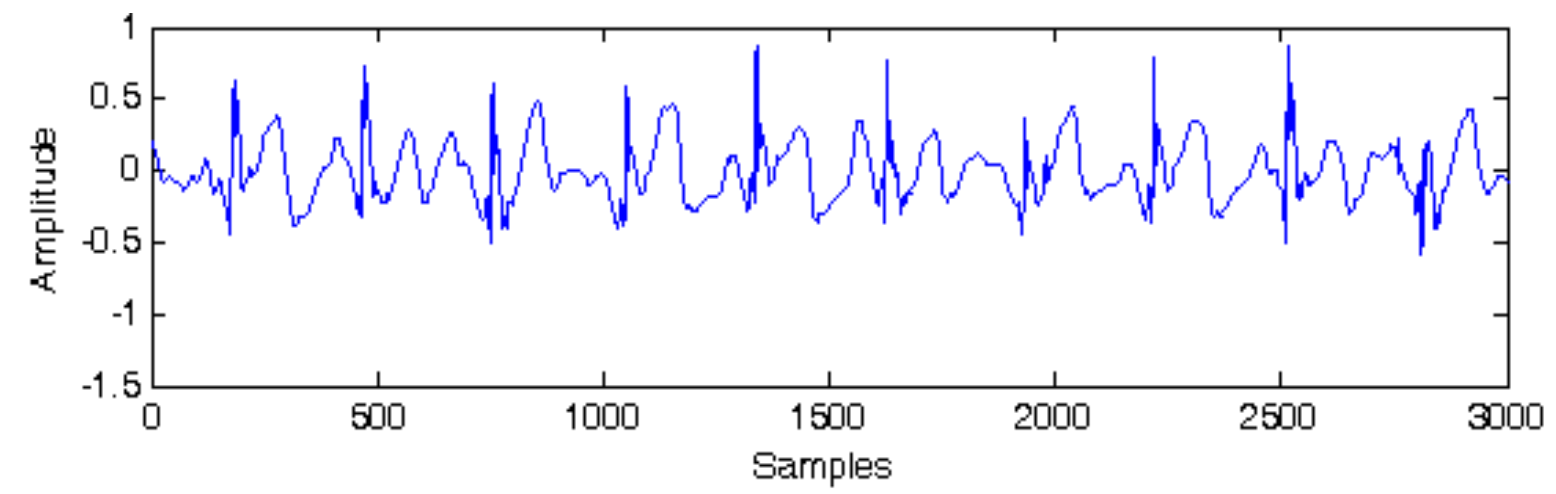

(c)

Fig.4. Result of noise removal from LVE signal; (a) ECG signal with noise, (b) noise removal using Sqtwolog, (c) noise reduction using Sqtwolog-HL

A similar experiment to that shown in Fig. 3 was repeated with different ECG signals as shown in Fig. 4. Fig. 4 repeats the sequence as in Fig. 3 that includes Fig. 3(a) signals with noise, Fig. 3(b) result of Sqtwolog denoising method and Fig. 3(c) result of Sqtwolog-HL denoising method. Fig. 3 (b) shows the removal of the high frequency noise using the 
denoising technique leaving low frequency noise, while Fig. 3 (c) shows the result without the low and high frequency noise removed by the combination of the denoising and high-pass/low-pass filter. Most of the important information is still present although some noise still corrupted the signal, since the spectrum of the noise overlapped with the ECG signal.

\section{CONCLUSION}

The combination of two wavelet technique; wavelet based denoising method and wavelet based high-pass/low-pass filter is able to produce better results than denoising methods applied on their own. The experimental results also show the capability of the proposed method to denoise both the low frequency and high frequency noise leaving behind the important information of the original signal.

\section{ACKNOWLEDGEMENTS}

This research work is supported by the (RAGS/1/2014/TK03/UPNM/2) research grant.

\section{REFERENCES}

[1] Arvinti B, Toader D, Costache M, Isar A. Electrocardiogram baseline wander removal using stationary wavelet approximations. In 12th International Conference Optimization of Electrical and Electronic Equipment, 2010, pp. 890-895

[2] Braunwald E. Heart disease: A textbook of cardiovascular medicine. Philadelphia: W. B. Saunders Company, 1997

[3] Bsoul A A R, Ji SY, Ward K, Najarian K. Detection of P, QRS and T components of ECG using wavelet transformation. In International Conference on Complex Medical Engineering, 2009, pp. 1123-1128

[4] Donoho D L. De-noising by soft-thresholding. IEEE Transactions on Information Theory, 1995, 41(3):613-627

[5] Donoho D L, Johnstone I M. Ideal spatial adaptation by wavelet shrinkage. Biometrika, $1994,81(3): 425-455$

[6] Hashim F R, Petropoulakis, L, Soraghan J, Safie S I. Wavelet based motion artifact 
removal for ECG signals. In Biomedical Engineering and Sciences, 2012, pp. 339-342

[7] Kirst M, Glauner B, Ottenbacher J. Using DWT for ECG motion artifact reduction with noise-correlating signals. In IEEE 33rd Annual International Conference, 2011, pp. 4804-4807 [8] Meyer Y., Roques S. Progress in wavelet analysis and applications: Proceedings of the International Conference "Wavelets and Applications" Toulouse, France-June 1992. Biarritz: Atlantica Séguier Frontières, 1993

[9] Nagendra H, Mukherjee S, Kumar V. Wavelet based nonlinear thresholding techniques for pre-processing ECG signals. International Journal of Biomedical and Advance Research, 2013, 4(8):534-544

[10] Percival D. B., Walden A. T. Wavelet methods for time series analysis. England: Cambridge University Press, 2006

[11] Surekha K S, Patil B P. ECG signal compression using hybrid 1D and 2D wavelet transform. In Science and Information Conference, 2014, pp. 3204-3208

[12] Thakor N V, Zhu Y S. Applications of adaptive filtering to ECG analysis: Noise cancellation and arrhythmia detection. IEEE Transactions on Biomedical Engineering, 1991, 38(8):785-794

[13] Zhe L, Jun N, Xin G. A denoising framework for ECG signal processing. In 6th International Conference Internet Computing for Science and Engineering, 2012, pp. 176-179 [14] Zhang W., Yang X.. Xu Z., An P., Liu Q., Lu Y. Advances on digital television and wireless multimedia communications. 9th International Forum on Digital TV and Wireless Multimedia Communication, IFTC 2012, Shanghai, China, November 2012, Proceedings. Berlin: Springer Verlag, 2012

[15] Nor M N M, Jailani R, Tahir N M, Yassin I M, Zairi I R, Rahmat H. EMG signals analysis of BF and RF muscles in autism spectrum disorder (ASD) during walking. International Journal on Advanced Science, Engineering and Information Technology, 2016, 6(5):793-798.

\section{How to cite this article:}

Hashim FR, Adnan J, Nk Daud NG, Mokhtar ASN, Rashidi AF, Rizman ZI. Electrocardiogram noise cancellation using wavelet transform. J. Fundam. Appl. Sci., 2017, 9(3S), 131-140. 